\title{
Trigeminal neuralgia and its management
}

\author{
Luke Bennetto', Nikunj K Patel ${ }^{2}$, Geraint Fuller ${ }^{3}$
}

$\overline{1 \text { Institute of Clinical Neurosciences, }}$ University of Bristol, Frenchay Hospital, Bristol BS161LE

${ }^{2}$ Department of Neurosurgery,

Frenchay Hospital

${ }^{3}$ Gloucester Royal Hospital,

Gloucester GL1 3NN

Correspondence to: G Fuller

geraint.fuller@glos.nhs.uk

BMJ 2007;334:201-5

doi: 10.1136/bmj.39085.614792.BE
Trigeminal neuralgia is a severe unilateral paroxysmal facial pain, often described by patients as the "the world's worst pain."

\section{Data sources and selection criteria}

We searched Medline, National Institute for Health and Clinical Excellence (NICE), and Cochrane databases using the terms "trigeminal neuralgia", "tic douloureux", and "facial pain". All related articles from the NICE and Cochrane databases were obtained. We searched Medline citation lists by title and abstract for relevant publications, including randomised control trials and review articles. The citation lists of these articles were hand searched for further relevant articles.

\section{How common is trigeminal neuralgia?}

The diagnosis is made by general practitioners in 27 per 100000 people each year ${ }^{1}$ in the United Kingdom. However, previous population based studies with a strict case definition estimated the rate to be $4-13$ per 100000 people each year. ${ }^{23}$ Almost twice as many women are affected as men. ${ }^{3}$ The incidence gradually increases with age and is rare below 40 .

\section{If it is uncommon why do I need to read this?}

The condition causes severe pain, which responds poorly to analgesics, but when recognised it can be

\begin{tabular}{l}
\hline SUMMARY POINTS \\
\hline Trigeminal neuralgia is a rare but characteristic pain \\
syndrome \\
Most cases are still referred to as idiopathic, although \\
many are associated with vascular compression of the \\
trigeminal nerve \\
A minority of cases are symptomatic of multiple sclerosis or \\
nerve compression by tumour \\
The condition is variable and patients may have just one \\
episode \\
Most patients respond well to drugs; carbamazepine is \\
usually the first line treatment \\
If drug treatment fails or is not tolerated, surgical \\
treatments are available \\
Ablative surgical treatments are associated with facial \\
sensory loss, almost no risk of severe complications or \\
death, and a high rate of pain recurrence; microvascular \\
decompression has a risk of severe complications or \\
death, albeit very low, and a lower relapse rate
\end{tabular}

treated. The pathophysiology of trigeminal neuralgia is becoming clearer. A wide range of medical and surgical treatments has been developed and introduced, usually without randomised clinical trials. As a result, uncertainty remains about how best to use the available treatments.

\section{How is the diagnosis made?}

Trigeminal neuralgia is a clinical diagnosis. The key feature is a sudden and severe lancinating pain, which usually lasts from a few seconds to two minutes, within the trigeminal nerve distribution, typically the maxillary or mandibular branches (fig 1). The pain is often evoked by trivial stimulation of appropriately named "trigger zones."

Occasionally the pain is so severe that it prevents eating or drinking. The nerves affected are usually stereotyped for a particular patient and lie within the sensory distribution of the trigeminal nerve. Box 1 lists the diagnostic criteria for the classic form of the disease.

In many cases the pain does not fit these criteria exactly because of a persistent ache between paroxysms or mild sensory loss. Such disease has been labelled as "atypical" or "mixed" trigeminal neuralgia. ${ }^{6}$ Patients with atypical disease are more likely to have symptomatic rather than idiopathic disease, and

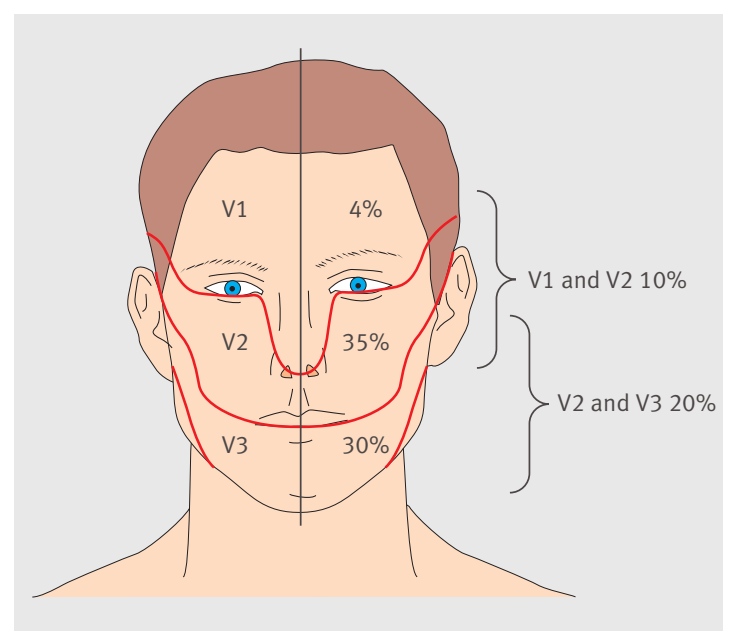

Fig 1 | Distribution of trigeminal neuralgia. ${ }^{3}$ In another $1 \%$ of patients it also affects all three divisions and rarely it can be bilateral (though paroxysms are not synchronous) 
Box 1 | Diagnostic criteria for classic trigeminal neuralgia ${ }^{4}$

- Paroxysmal attacks of pain lasting from a fraction of a second to two minutes that affect one or more divisions of the trigeminal nerve

- Pain has at least one of the following characteristics intense, sharp, superficial, or stabbing precipitated from trigger areas or by trigger factors

- Attacks are similar in individual patients

- No neurological deficit is clinically evident

- Not attributed to another disorder

Box 2 | Evidence that vascular compression commonly causes trigeminal neuralgia ${ }^{510}$

- An aberrant loop of artery, or less commonly vein, is found to be compressing the root entry zone of the trigeminal nerve in $80-90 \%$ of patients at surgery

- The trigeminal nerve is demyelinated next to the compressing vessel

- Eliminating the compression by surgery provides long term relief in most patients

- Intraoperative assessments report immediate improvement in trigeminal conduction on decompression

- Sensory function recovers after decompression

- Other causes, such as compression by tumours or the demyelinating plaques of multiple sclerosis, produce similar lesions of the root entry zone of the trigeminal nerve

they are often more refractory to treatment ${ }^{6}$ than those with classic trigeminal neuralgia. ${ }^{7}$ Atypical trigeminal neuralgia should not be confused with atypical facial pain (table 1).

\section{What causes trigeminal neuralgia?}

Increasing evidence (box 2) suggests that $80-90 \%$ of cases that are technically still classified as idiopathic are caused by compression of the trigeminal nerve (fig 2) close to its exit from the brainstem by an aberrant loop of artery or vein..$^{510}$

Importantly, compression is of the root entry zone, where axons are coated with central nervous system myelin, rather than peripheral nerve myelin. Similarly, vascular compression of the facial and glossopharyngeal nerves is thought to be responsible for most cases of hemifacial spasm and glossopharyngeal neuralgia, respectively. ${ }^{10 \mathrm{w} 1}$

Less than $10 \%$ of patients will have symptomatic disease associated with an identifiable cause other than a vascular compressive lesion-usually a benign tumour or cyst ${ }^{101}$-or multiple sclerosis. About 1-5\% of patients with multiple sclerosis develop trigeminal neuralgia. ${ }^{5}$ See bmj.com for an explanation of why trigeminal neuralgia is paroxysmal.

\section{Most facial pain is not trigeminal neuralgia}

Other causes of facial pain are much more common than trigeminal neuralgia. This can often lead to delay in diagnosis as patients see dentists and doctors who consider more common alternatives first.

Common causes of facial pain are usually straightforward to eliminate clinically or after dental examination (table 1); rarer alternatives may evade consideration (see table 2 on bmj.com). Many alternatives affect the forehead only, which is rare for trigeminal neuralgia, so trigeminal neuralgia affecting the forehead should be diagnosed with caution. Although tearing and other autonomic features can occur in trigeminal neuralgia, these features combined with forehead pain should prompt consideration of the trigeminal autonomic cephalalgias (for example, cluster headache). ${ }^{4}$

\section{What investigations are needed?}

Investigations are done to:

- Clarify the differential diagnosis; for example, by taking dental $\mathrm{x}$ rays

- Investigate whether there is an identifiable cause of the disease, particularly with a view to surgical cure. This is best done using magnetic resonance imaging.

As $5-10 \%{ }^{11}$ of cases are caused by tumours, multiple sclerosis, abnormalities of the skull base, or arteriovenous malformations the threshold for magnetic resonance imaging should be low. A brain scan should be obtained in younger patients; those with atypical clinical features, including sensory loss or a dull burning pain between paroxysms; and patients who do not respond to initial medical therapy.

With recent improvements in magnetic resonance imaging techniques (see details of the available techniques and fig 3 on bmj.com), vascular compression is being demonstrated radiologically in increasing numbers of patients with trigeminal neuralgia. Studies where radiologists are blinded to the side of the pain have produced good results in terms of predicting subsequent surgical findings and response to surgery. ${ }^{12 w 6}$ Postmortem studies have found vessels in contact with the trigeminal nerve in $3-12 \% 0^{5}$ of asymptomatic

Table 1 | Common conditions that are usually easy to distinguish from trigeminal neuralgia ${ }^{489}$

\begin{tabular}{|c|c|}
\hline Diagnosis & Important features \\
\hline Dental infection or cracked tooth & Well localised to tooth; local swelling and erythema; appropriate findings on dental examination \\
\hline Temporomandibular joint pain & Often bilateral and may radiate around ear and to neck and temples; jaw opening may be limited and can produce an audible click \\
\hline $\begin{array}{l}\text { Persistent idiopathic facial pain (previously } \\
\text { "atypical facial pain") }\end{array}$ & $\begin{array}{l}\text { Often bilateral and may extend out of trigeminal territory; pain often continuous, mild to moderate in severity, and aching or throbbing in } \\
\text { character }\end{array}$ \\
\hline Migraine & Often preceded by aura; severe unilateral headache often associated with nausea, photophobia, phonophobia, and neck stiffness \\
\hline Temporal arteritis & $\begin{array}{l}\text { Common in elderly people; temporal pain should be constant and often associated with jaw claudication, fever, and weight loss; temporal } \\
\text { arteries may be firm, tender, and non-pulsatile on examination }\end{array}$ \\
\hline
\end{tabular}




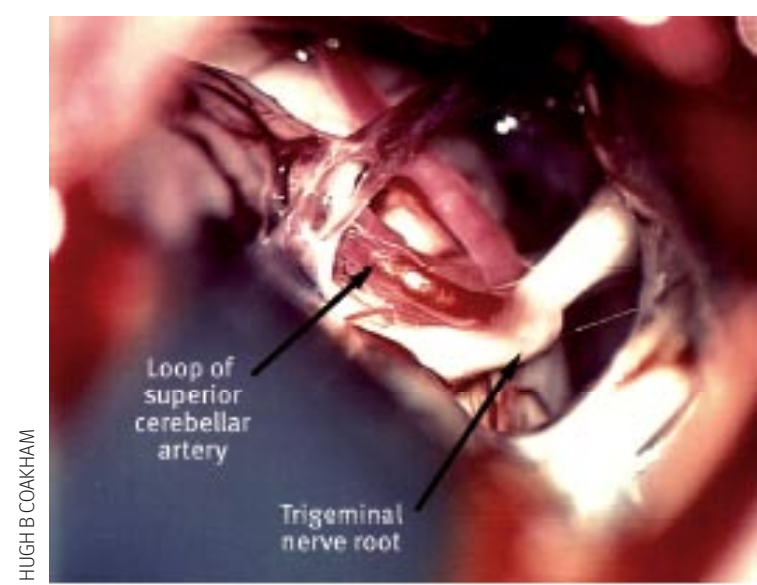

Fig 2 | Compression of the trigeminal nerve root by an aberrant loop of the superior cerebellar artery

patients. Thus, currently these imaging techniques should be used to explore surgical treatment options in clinically diagnosed trigeminal neuralgia, rather than in making a diagnosis.

\section{What is the natural course of trigeminal neuralgia?}

Because the severity of the pain demands intervention, no studies of the natural course of the disease are available. One study collected information from linked primary and secondary care records over a 40 year period. ${ }^{3}$ They found that $29 \%$ of patients had only one episode of pain, 19\% had two, $24 \%$ had three, and $28 \%$ had four to 11 . Each episode lasted from one day to four years (median 49 days). After the first episode $65 \%$ of patients had a second within five years, though in $23 \%$ the gap was more than 10 years. Similar ranges of delay were seen from the second to the third event. This highlights the wide spectrum from single to fre-

Box $3 \mid$ Problems associated with studies of trigeminal neuralgia

\section{Related to the condition}

- Condition rare

- Condition variable, with spontaneous remission and relapse

- Condition has uncertain natural history

- Condition is a clinically defined syndrome with no gold standard diagnosis

- The pain is so severe that placebo control studies are considered unethical

\section{Related to the study}

- Diagnostic criteria variably interpreted and applied

- Lack of standardised outcome measures

- Quality of life rarely measured

- Open unblinded non-randomised studies predominate

- Based on case series, usually from tertiary referral centres

\section{Related to treatment}

- Carbamazepine, the standard treatment, has a long half life and induces liver enzymes, making crossover studies difficult

- Most drugs used are out of patent, which limits funding opportunities

Related to surgery ${ }^{16}$

- Difficult to achieve blinding or use sham operations

- Different types of risks are being compared with different procedures (see text) quent episodes, with each episode being of variable duration.

Most of the case series reporting surgical and other treatments for trigeminal neuralgia are from tertiary care centres and therefore represent the most severely affected patients.

\section{What are the best medical treatments?}

Drug treatments for trigeminal neuralgia have been the subject of several Cochrane systematic reviews. ${ }^{13-15}$ These reviews bring together the small number of trials available in a condition that poses difficulties for study design (box 3). Perhaps unsurprisingly the evidence is mostly weak.

Available evidence shows that carbamazepine is the drug of choice (table 3 on bmj.com). ${ }^{15}$ Many patients develop adverse effects, however, though most can continue taking the drug. If the patient responds well, a controlled release preparation can be substituted and the dose can gradually be reduced.

What is less clear is what to do if a patient is intolerant or allergic to carbamazepine, or if the drug is ineffective. In the absence of clear evidence of the effectiveness of other drugs, the choice between other agents can be made on the basis of adverse effects and ease of use (table 3 on bmj.com).

\section{If carbamazepine has adverse effects (table on bmj.com)} Oxcarbazepine is a prodrug of carbamazepine that is often better tolerated; it provides a logical, ${ }^{18}$ if largely unproved, ${ }^{17}$ alternative when carbamazepine has provided pain relief but has had unacceptable adverse effects. The risk of allergic crossreactivity between carbamazepine and oxcarbazepine is about 25\%, so oxcarbazepine is best avoided in carbamazepine allergy.

Gabapentin is effective and widely used for neuropathic pain, though it lacks evidence in trigeminal neuralgia. ${ }^{14}$ Use of gabapentin therefore relies on the similarities between trigeminal neuralgia and other neuropathic pain, rather than their obvious differences. Familiarity with use in other neuropathic pain has led many clinicians to choose this as second line for trigeminal neuralgia.

Lamotrigine and baclofen have been suggested as alternative second line agents on the basis of small studies in trigeminal neuralgia (see table 2 on bmj.com). In practice, lamotrigine needs to be titrated over many weeks and has limited value in severe pain. Other drugs to consider are phenytoin, clonazepam, valproate, mexiletine, and topiramate. ${ }^{17}$

\section{If carbamazepine is ineffective}

If pain relief is incomplete with carbamazepine options include adding a second agent or switching drugs. Similar considerations regarding choice of second line agent discussed above will apply.

Failure of medical therapy should prompt a review of the diagnosis ${ }^{19}$ (see table 1). If pain control cannot be achieved or drugs cause unacceptable adverse effects, surgical options should be considered. 


\section{ADDITIONAL EDUCATIONAL RESOURCES FOR PATIENTS}

- Zakrzewska JM. Insights-facts and stories behind trigeminal neuralgia. Gainesville, FL:

Trigeminal Neuralgia Association, 2006

- Trigeminal Neuralgia Association UK, Bromley BR2 9XS (URL: www.tna.org.uk;

Tel: +44 020 84629122)-Provides excellent information and patient support groups

- Trigeminal Neuralgia Association, Gainesville, FL 32605-6402, USA (URL: www.

tna-support.org; Tel: +01 8009233608 or +01 352331 7009) -Provides excellent information and patient support groups

- Brain and Spine Foundation, London SW9 6EJ (URL: WwW.brainandspine.org.uk; Tel: +44 0207793 5900) - Provides patient information and helpline

\section{RESEARCH QUESTIONS}

- What is the natural history of trigeminal neuralgia?

-Why do some patients with vascular compression of the trigeminal nerve get the disease and others not?

- Many drugs have some effect, but which is the best drug to use? A pragmatic comparative study is needed to clarify these choices.

- Can the response to drugs and to different surgical interventions be predicted on clinical or radiological grounds?

- Which ablative procedure is most effective for the fewest adverse effects?

- How do ablative procedures compare with microvascular decompression?

\section{What are the surgical treatment options?}

Two types of surgical procedure are available (table 4 on bmj.com):

- Microvascular decompression, where the posterior fossa is explored and the compressing vessel and trigeminal nerve root are separated

- Ablative treatments that lesion the trigeminal nerve in different ways.

As with most surgical procedures the literature comprises mainly case series. Such series are difficult to compare (see box 3), as they vary in the populations of patients studied; diagnostic criteria, outcome measures, and follow-up methods used; and the presentation of the data. ${ }^{72}$ Despite this, broadly comparable high quality studies show relatively consistent responses to each procedure (fig 4 on bmj.com).

Predicting outcome for individual patients with confidence is difficult. Case series suggest that patients with classic trigeminal neuralgia, evidence of vascular compression, shorter duration of disease, and no previous surgery respond better to all treatment options. ${ }^{2021}$ In such patients, microvascular decompression can be considered the "gold standard" surgical procedure, and it offers the best long term cure rates. Outcome also varies with the case load of operating surgeons. ${ }^{22}$

Deciding on which procedure to use involves choosing between two different types of risk. All procedures have a high initial response rate, except for stereotactic radiosurgery, which usually takes maximum effect at one to two months (fig 4 on bmj.com). ${ }^{23}$ Microvascular decompression has the best chance of long term pain relief, with a very low risk of facial sensory loss and other minor complications; however, it has a small risk of death (around $0.4 \%{ }^{24}$ ). These risks vary according to other comorbidities that alter operative risk. In contrast, ablative procedures are less effective in the long term and more likely to produce facial numbness and other minor complications; indeed their effectiveness is often greatest when this is the case. ${ }^{7}$ They have a much lower risk of death or major complication, however, and are used to a greater extent in patients with high operative risk or as a partially diagnostic procedure in atypical disease. Gamma knife stereotactic radiosurgery has recently been approved ${ }^{23}$ by the UK's National Institute for Health and Clinical Excellence for treatment of trigeminal neuralgia, but access to this treatment is limited.

Making a decision between these options depends on the patient's perception of the two very different types of risk. Patients need to be well informed of the options, the related risks, and the likely outcomes to make such a decision. After having microvascular decompression most patients said they wish they had undergone the procedure sooner. ${ }^{25}$ Choosing between different ablative procedures, which seem to have similar effects, may be influenced by factors such as the range of adverse effects and the way in which the procedure is undertaken (see table 4 on bmj.com).

The patient with trigeminal neuralgia who faces these difficult choices needs to be provided with the best available information and support from the doctors and surgeons involved in their care. They may find information from patient associations and other publications useful.

\section{Patient's perspective}

It was 1992 when I developed pain of a "shock nature" that seemed to run around the top of my teeth on the right side. I saw the dentist who said it was nothing to do with my teeth. My doctor put me on tegretol, which made me feel like a "zombie" and I became unsteady on my feet. By now the pain was travelling in towards the brain and down towards the jaw. It was excruciating, and the only way I could "hold" the pain was to grit my teeth together very hard until the pain died away. Sleep was not interrupted, but on waking the slightest movement of the mouth would set the spasms off again, so I didn't eat or talk much. I lost a lot of weight. I went to see a neurosurgeon who advised me to have an operation to "cuff" the responsible nerve, and despite the risks I agreed as the pain was intolerable. After the operation the pain had gone. I was alive again.

Ten years later the pain returned without warning. I saw the neurosurgeon again and this time I had a "radiofrequency lesion" of my trigeminal nerve. I am at present pain free and enjoying life as much as an 86 year old man can.

Contributors: LB and GF reviewed the literature and wrote the article. NKP reviewed and revised the article. GF is guarantor.

Competing interests: None declared.

1 Hall GC, Carroll D, Parry D, McQuay HJ. Epidemiology and treatment of neuropathic pain: the UK primary care perspective. Pain 2006;122:156-62.

2 MacDonald BK, Cockerell OC, Sander JW, Shorvon SD. The incidence and lifetime prevalence of neurological disorders in a prospective community-based study in the UK. Brain 2000;123:665-76.

3 Katusic S, Beard CM, Bergstralh E, Kurland LT. Incidence and clinical features of trigeminal neuralgia, Rochester, Minnesota, 1945-1984. Ann Neurol 1990;27:89-95. 
4 Headache Classification Subcommitee of the International Headache Society. The international classification of headache disorders. 2nd edition. Cephalalgia 2004;24:1-150.

5 Nurmikko TJ, Eldridge PR. Trigeminal neuralgia-pathophysiology, diagnosis and current treatment. Br/Anaesth 2001;87:117-32.

6 Zakrzewska JM, Jassim S, Bulman IS. A prospective, longitudinal study on patients with trigeminal neuralgia who underwent radiofrequency thermocoagulation of the Gasserian ganglion. Pain 1999; $79: 51-8$

7 Lopez BC, Hamlyn PJ, Zakrzewska JM. Systematic review of ablative neurosurgical techniques for the treatment of trigeminal neuralgia. Neurosurgery 2004;54:973-82.

8 Zakrzewska JM. Facial pain: neurological and non-neurological. J Neurol Neurosurg Psychiatry 2002;72(suppl 2):ii27-32.

9 Zakrzewska JM. Diagnosis and differential diagnosis of trigeminal neuralgia. Clin I Pain 2002;18:14-21.

10 Love S, Coakham HB. Trigeminal neuralgia: pathology and pathogenesis. Brain 2001;124:2347-60.

11 Cheng TM, Cascino TL, Onofrio BM. Comprehensive study of diagnosis and treatment of trigeminal neuralgia secondary to tumors. Neurology 1993;43:2298-302.

12 Patel NK, Aquilina K, Clarke Y, Renowden SA, Coakham HB. How accurate is magnetic resonance angiography in predicting neurovascular compression in patients with trigeminal neuralgia? A prospective, single-blinded comparative study. Br J Neurosurg 2003;17:60-4.

13 Wiffen P, Collins S, McQuay H, Carroll D, Jadad A, Moore A. Anticonvulsant drugs for acute and chronic pain. Cochrane Database Syst Rev 2005;(3):CD001133.

14 Wiffen PJ, McQuay HJ, Edwards JE, Moore RA. Gabapentin for acute and chronic pain. Cochrane Database Syst Rev 2005;(3): CD005452.

15 Wiffen PJ, McQuay HJ, Moore RA. Carbamazepine for acute and chronic pain. Cochrane Database Syst Rev 2005;(3):CD005451.

16 Zakrzewska JM, Lopez BC. Quality of reporting in evaluations of surgical treatment of trigeminal neuralgia: recommendations for future reports. Neurosurgery 2003;53:110-20.

17 Zakrzewska JM, Lopez BC. Trigeminal neuralgia. Clin Evid 2005;1669 77.

18 Canavero S, Bonicalzi V. Drug therapy of trigeminal neuralgia. Expert Rev Neurother 2006;6:429-40.

19 Sato J, Saitoh T, Notani K, Fukuda H, Kaneyama K, Segami N. Diagnostic significance of carbamazepine and trigger zones in trigeminal neuralgia. Oral Surg Oral Med Oral Pathol Oral Radiol Endod 2004:97:18-22.

20 Lopez BC, Hamlyn PJ, Zakrzewska JM. Stereotactic radiosurgery for primary trigeminal neuralgia: state of the evidence and recommendations for future reports. I Neurol Neurosurg Psychiatry 2004;75:1019-24

21 Li ST, Pan Q, Liu N, Shen F, Liu Z, Guan Y. Trigeminal neuralgia: what are the important factors for good operative outcomes with microvascular decompression. Surg Neurol 2004;62:400-4.

22 Kalkanis SN, Eskandar EN, Carter BS, Barker FG. Microvascular decompression surgery in the United States, 1996 to 2000: mortality rates, morbidity rates, and the effects of hospital and surgeon volumes. Neurosurgery 2003;52:1251-61.

23 Lim JNW, Ayiku L. The clinical efficacy and safety of stereotactic radiosurgery (gamma knife) in the treatment of trigeminal neuralgia. London: National Institute for Health and Clinical Excellence, 2004. www.nice.org.uk/guidance/IPG85

24 Ashkan K, Marsh $\mathrm{H}$. Microvascular decompression for trigeminal neuralgia in the elderly: a review of the safety and efficacy. Neurosurgery 2004:55:840-8.

25 Zakrzewska JM, Lopez BC, Kim SE, Coakham HB. Patient reports of satisfaction after microvascular decompression and partial sensory rhizotomy for trigeminal neuralgia. Neurosurgery 2005;56:1304-11.

\section{When I use a word} Colourful metals

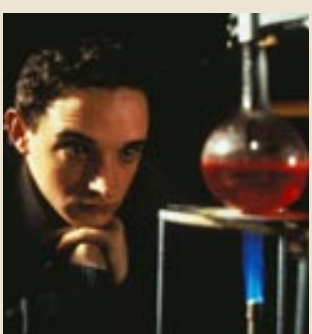

The film The Young Poisoner's Handbook, was loosely based on the life of thallium poisoner Graham Young
In 1859 Gustav Kirchhoff and Robert Bunsen invented a method for detecting the light spectra that substances produce when heated. They used the gas burner that Bunsen had invented, whose flame is very hot with little luminescence. This led to the discovery of elements whose names reflected the colour of the resultant light: - Caesium-(discovered by Kirchhoff and Bunsen in 1860) from the Latin caesius, grey-blue, usually referring to the eyes

- Rubidium-(Kirchhoff and Bunsen, 1861) from Latin rubidus, red, describing a facial flush

- Thallium-(William Crookes, 1861) from Greek thallos, a young olive green shoot

-Indium-(Ferdinand Reich and Hieronymus Richter, 1863) from Greek indikon, indigo.

Chlorine gas is green (Greek chloros), and iodine vapour is violet (Greek ion). Platinum shines like silver (Spanish plata), and orpiment (aurum pigmentum, arsenic trisulphide) gleams like gold; the Greek for orpiment was arsenikon (Hebrew zarnik). Gold (Arabic zarqun) also gives us zirconium, from the golden mineral zircon. Like chromium (Greek chroma, colour), rhodium (rhodon, a rose), and praseodymium (prasios, leek green, also vomitus), vanadium may get its name from its attractively coloured salts-Vanadis was the beautiful Norse goddess Freya or one of her attendants. One of the most colourful elements, iridium, is named after the rainbow (Greek iris), and pale bismuth (German Wismut) may mean "white mass."

When Alexander Litvinenko, a former KGB officer and an outspoken critic of Vladimir Putin, became ill while investigating the murder of the Russian journalist Anna Politkovskaya, thallium poisoning was suspected. The cardinal features are nausea, vomiting, diarrhoea, a painful ascending neuropathy, skin rashes, and alopecia. Agatha Christie described thallium poisoning in her novel The Pale Horse (Collins, 1961), and Dennis Sanders and Len Lovallo, in The Agatha Christie Companion (W H Allen, 1985), described three cases in which the diagnosis was made by people who had read the book and recognised the symptoms. In 1975 a woman wrote to Christie from a Latin American country, telling her that, having read The Pale Horse, she had saved the life of a man whose wife was poisoning him.

In 1977 a London nurse, Marsha Maitland, diagnosed thallium poisoning in a 19 month old baby from Qatar. An earlier case is the best known. In 1971, workers at the Hadlands Photographic Equipment works in Bovingdon, Hampshire, developed illnesses thought to be infectious, attributed to the "Bovingdon bug"; some died. Dr Hugh Johnson, a forensic expert, remembering Christie's novel, suspected thallium poisoning and fingered Graham Frederick Young, who worked at Hadlands. Thallium and other poisons were found in his flat. After the jury found him guilty, it was revealed that he had not long before been released from Broadmoor, after serving nine years of a 15 year sentence for the attempted murders of his father, sister, and a friend; he probably also murdered his mother.

In 1996 a Chinese girl called Zhu Ling developed an illness that was diagnosed first as GuillainBarré syndrome and then as acute disseminated encephalomyelitis (BMJ 1996;312:318-9). When a friend sent a message on the internet asking for help many respondents suggested thallium poisoning, which was confirmed.

In 2005 an unnamed Japanese girl was accused of poisoning her mother with thallium. She had read Anthony Holden's book, The St Albans Poisoner: The Life and Crimes of Graham Young (Hodder \& Stoughton, 1974), had seen The Young Poisoner's Handbook (1995), a film based on Young's life, and had read The Pale Horse.

However, thallium was not the green toxin that killed Litvinenko, but a red herring. The poison was polonium, which is another story. Jeff Aronson clinical pharmacologist, Oxford jeffrey.aronson@clinpharm.ox.ac.uk 